\title{
Activation of haemostasis by exercise, mental stress and adrenaline: effects on platelet sensitivity to thrombin and thrombin generation
}

\author{
N. Håkan WALLÉN, Alison H. GOODALL*, Nailin LI and Paul HJEMDAHL \\ Department of Clinical Pharmacology, Karolinska Hospital, S-17I 76 Stockholm, Sweden, and *Division of Chemical Pathology, \\ University of Leicester, Glenfield Hospital, Groby Road, Leicester LE3 9QP, U.K.
}

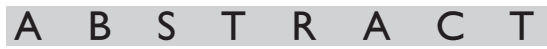

\begin{abstract}
Stress-induced activation of haemostasis may be involved in the triggering of acute coronary syndromes. We compared the effects of mental stress, dynamic exercise and adrenaline infusion on platelet sensitivity to thrombin using flow-cytometric analysis of platelet fibrinogen binding in whole blood, and platelet aggregability using filtragometry ex vivo, in healthy volunteers. Furthermore, we assessed thrombin generation [prothrombin fragment $\mathrm{I}+2(\mathrm{FI}+2)$ and thrombin-antithrombin complexes in plasma] and thrombin activity (fibrinopeptide $A$ in plasma). Exercise (bicycle ergometry) enhanced thrombin-induced platelet fibrinogen binding $(P<0.05)$ and platelet aggregability $(P<0.01)$, and elevated $\mathrm{FI}+2$, thrombin-antithrombin complexes and fibrinopeptide $A(P<0.05$ for all three). Adrenaline infusion enhanced thrombin-induced platelet fibrinogen binding and platelet aggregability $(P<0.05)$, and elevated thrombin-antithrombin complexes $(P<0.05)$, whereas $\mathrm{FI}+2$ and fibrinopeptide $A$ levels were not significantly affected. Mental stress increased platelet sensitivity to high concentrations of thrombin only, and produced small increases in levels of thrombin-antithrombin complexes. Time control experiments showed no important changes with repeated measurements during rest. Platelet responses to exercise and adrenaline were reversible, with recovery $60 \mathrm{~min}$ later. Thus, heavy exercise and high levels of adrenaline reversibly increased platelet aggregability and platelet sensitivity to thrombin, and enhanced thrombin formation; the effects were most pronounced during exercise. Mental stress only weakly affected these parameters.
\end{abstract}

\section{INTRODUCTION}

External stressors, such as mental stress and physical exertion, may be involved in the triggering of acute coronary syndromes via activation of haemostasis $[1,2]$. Platelet activation has been shown after mental stress [3], and physical exercise $[4,5]$ and surges of catecholamines in plasma significantly increase platelet aggregability and platelet secretion in healthy humans in vivo, as evidenced by studies of responses to infused adrenaline [6] or noradrenaline [7]. However, comparative studies are few. It may be hypothesized that the coagulation system is also activated after 'stress'. Increased formation of thrombin after physical exercise has been shown previously $[8,9]$ but data on effects of mental stress and catecholamines on the generation of thrombin are scarce. Thrombin is not only central to coagulation but is also a very potent platelet agonist, which may influence platelet function in vivo.

In the present study we therefore compared the effects

Key words: flow cytometry, filtragometry, platelet function, thrombin.

Abbreviations: F1 +2, prothrombin fragment 1+2; FITC, fluorescein isothiocyanate; FPA, fibrinopeptide A; TAT, thrombinantithrombin complexes.

Correspondence: Dr Håkan Wallén. 
of physical exercise, mental stress and adrenaline infusion on various aspects of platelet function and thrombin generation in vivo in young healthy volunteers. Platelet aggregability was assessed using filtragometry ex vivo, and platelet sensitivity to thrombin was measured by flow-cytometric analysis of platelet fibrinogen binding in whole blood. Thrombin generation was assessed by measurements of the levels of prothrombin fragment $1+2(\mathrm{~F} 1+2)$ and thrombin-antithrombin complexes (TAT) in plasma. In addition, we measured thrombin activity by measurements of fibrinopeptide A (FPA) in plasma.

\section{MATERIALS AND METHODS}

\section{Subjects and procedures}

The study was approved by the Ethics Committee of the Karolinska Hospital, and the subjects gave their informed consent before participating. Eleven males, mean age 28 years (range 24-35 years), participated in the study. The volunteers were healthy, non-smokers, and had easily accessible antecubital veins in both arms to ensure clean venepunctures. They were instructed not to take plateletactive drugs for at least 14 days before the experiments, which were performed on four separate occasions.

The experiments were always performed between 08.00 and $12.00 \mathrm{~h}$. Blood sampling and filtragometry measurements were performed after $60 \mathrm{~min}$ of rest in a semi-reclined position, during or after the interventions (exercise, mental stress or intravenous infusions of adrenaline; see below), and again after $60 \mathrm{~min}$ of rest, i.e. recovery. Time control experiments were performed on the fourth occasion.

Blood pressure and heart rate were measured by an Exercise Monitor 1160 (Critikon Inc., Tampa, FL, U.S.A.).

The exercise test was carried out on a bicycle ergometer (Siemens AB, Solna, Sweden), starting at a workload of $30 \mathrm{~W}$ with increments of $10 \mathrm{~W} / \mathrm{min}$ and terminated upon exhaustion. Fatigue during the exercise test was estimated according to a 20-graded category-ratio scale [10]. Blood sampling and filtragometry measurements were performed within $30 \mathrm{~s}$ of termination of the exercise test.

Adrenaline (ACO, Solna, Sweden; diluted in cold saline with $0.1 \mathrm{mg} / \mathrm{ml}$ ascorbic acid added as antioxidant) was infused by means of a pump (Braun perfusor) via an indwelling intravenous cannula inserted in an easily accessible vein at the ankle, at 0.1 and $0.4 \mathrm{nmol} \cdot \mathrm{min}^{-1} \cdot \mathrm{kg}^{-1}$, and with each dose step lasting $20 \mathrm{~min}$. The dose was chosen to reproduce the conditions previously used to demonstrate effects of adrenaline by flow cytometry [11]. Blood sampling and filtragometry measurements were performed after the high dose only.

Mental stress was induced by a modified videotaped version of Stroop's colour word conflict test [12]. Haemodynamic responses to this mental stress test have been shown to be reproducible, and to reach a steady state after 8-10 min [13]. Filtragometry measurements and sampling were carried out after $15 \mathrm{~min}$ of mental stress, with the test running.

Time control experiments were performed with the subjects resting in a semi-reclined position. Measurements and sampling were carried out after 60, 100 and $160 \mathrm{~min}$ of rest.

\section{Venepuncture and blood sampling}

An anaesthetic cream $\left(\mathrm{EMLA}^{\circledR}\right.$, Astra, Södertälje, Sweden) was applied on the skin over the antecubital veins to minimize discomfort from the venepunctures. Filtragometry measurements, and blood sampling for blood cell counts (i.e. platelet counts, platelet volume and haematocrit) and plasma catecholamine measurements, were carried out by venepuncture of an antecubital vein using a 19-gauge butterfly needle. Simultaneously, blood for determination of thrombin markers and flow-cytometric analyses was drawn from an antecubital vein in the contralateral arm, using a 21-gauge needle connected to vacutainer tubes containing appropriate additives (see below). Samples were always taken in the following order: two 10-ml tubes for thrombin markers [containing an anticoagulant mixture of $10 \mathrm{mmol} / \mathrm{l} \mathrm{EDTA,} 1 \mu \mathrm{mol} / 1$ PPACK (a selective thrombin inhibitor; D-Phe-Pro-Arg chloromethyl ketone hydrochloride) and $14 \mu \mathrm{g} / \mathrm{ml}$ aprotinin; final concentrations in the sample], one $5-\mathrm{ml}$ spare tube and one 5-ml tube for flow cytometry (both tubes containing sodium citrate giving a final concentration of $0.38 \%$ ). All venepunctures were performed without stasis.

\section{Filtragometry ex vivo}

Equipment and procedures for measurements with the filtragometry technique have been described in detail previously $[14,15]$. The technique has been shown to measure platelet aggregates ex vivo in blood continuously drawn from an antecubital vein. Each reading requires a new venepuncture. The continuously drawn blood, which is anticoagulated by a heparin infusion (final concentration 5 i.u. $/ \mathrm{ml}$ ) in the tubing, passes through a nickel filter $(2.0 \times 0.2 \mathrm{~mm})$ with a pore size of $20 \mu \mathrm{m}$. Occlusion of the filter is assessed by pressure transducers, and the time until $25 \%$ filter occlusion is measured $\left(t_{\mathrm{A}}\right.$; this may range from $60-800 \mathrm{~s}$ at rest). $t_{\mathrm{A}}$ reflects platelet aggregability inversely, i.e. rapid filter occlusion with a low $t_{\mathrm{A}}$ value indicates high platelet aggregability. The apparatus may influence platelet function due to shear forces when blood passes through the filter, and the addition of heparin may contribute to platelet aggregate formation, but these possible effects are standardized, and equal in all measurements. 


\section{Flow-cytometric assay}

\section{Reagents}

The platelets were identified by monoclonal antibodies against GPIb $\alpha$ (CD42b; RFGP37) and GPIIb-IIIa (CD41, RFGP56), coupled to fluorescein isothiocyanate (FITC) at a FITC to protein ratio of 3-4:1 (Cymbus Biotechnology Ltd, Chandlersford, Hants, U.K.). Both monoclonal antibodies were raised in Dr Goodall's laboratory [16]. Platelet-bound fibrinogen was detected with polyclonal rabbit anti-human fibrinogen coupled to FITC (R $\alpha f g n-F I T C)$, purchased from DAKO (Dakopatts, Stockholm). All antibodies were used at optimum concentrations that gave maximum fluorescence with minimum non-specific binding.

The dilution buffer was always Hepes-buffered saline (145 mmol/l NaCl, $5 \mathrm{mmol} / \mathrm{K} \mathrm{KCl}, 1 \mathrm{mmol} / \mathrm{l} \mathrm{MgSO}_{4}$ and $10 \mathrm{mmol} / 1 \mathrm{Hepes}$; $\mathrm{pH} 7.4)$ that had been passed through a $0.22 \mu \mathrm{m}$ filter. All reagents were analytical grade or above. ADP, human $\alpha$-thrombin and GPRP (Gly-Pro-Arg-Pro) were all purchased from Sigma Chemical Co. (St. Louis, MO, U.S.A.).

\section{Flow-cytometric analysis procedure}

The flow-cytometric analysis of platelets from whole blood has been described previously [11,17]. Within 10 min of collection, $5 \mu$ l of whole blood, anticoagulated with sodium citrate (final concentration $0.38 \%$ ), was pipetted into polystyrene tubes containing $50 \mu \mathrm{l}$ of Hepes-saline buffer, $5 \mu$ of the appropriate agonist (final concentrations of thrombin $0.01-0.32 \mathrm{NIH}$ units $/ \mathrm{ml}$ or ADP $10^{-7}-10^{-5} \mathrm{~mol} / \mathrm{l}$ ) and $5 \mu \mathrm{l}$ of an appropriate dilution of antibody. When thrombin was used as the agonist, $2 \mu \mathrm{l}$ of GPRP (final concentration $0.8 \mathrm{mmol} / \mathrm{l}$ ) was added to the tubes in order to inhibit fibrin cross-linking [18]. The samples were incubated without stirring for $20 \mathrm{~min}$ at room temperature, and then mixed with $500 \mu \mathrm{l}$ of formyl saline $[0.2 \%(\mathrm{v} / \mathrm{v})$ formaldehyde in $0.9 \%(\mathrm{w} / \mathrm{v}) \mathrm{NaCl}]$ to stop further activation. The samples were then diluted $1: 7$ and analysed 20 min later in a Coulter Epics XL flow cytometer (Coulter Electronics Ltd, Luton, U.K.). The flow cytometer was aligned daily with $10 \mu \mathrm{m}$ 'Immunocheck' and 'Standard Brite' beads (Coulter Immunology) to calibrate light scatter and fluorescence parameters respectively. The platelet population was identified by its light-scatter characteristics and enclosed in an electronic bit map. Platelets (5000 per sample) were analysed, and the results represent the means of duplicate samples. Samples from each subject were labelled with RFGP37-FITC or RFGP56-FITC to confirm that more than $97 \%$ of the analysed particles in each were GPIb $\alpha$ and GPIIb-IIIa positive. Levels of GPIb $\alpha$ and GPIIbIIIa per platelet were also measured in these samples.

The percentage of platelets positive for the marker and the mean fluorescence intensity (MFI) for each sample were used to calculate a binding index for the platelet activation marker from the following equation [11]:

$$
\text { Binding index }=(\% \text { positive } \times \text { MFI }) / 100
$$

\section{Thrombin markers}

Blood was collected into 10-ml vacutainer tubes (Becton Dickinson), pre-filled with an anticoagulant mixture containing EDTA, aprotinin and PPACK (see above), purchased from Byk Sangtec Diagnostica, Dietzenbach, Germany. Blood samples were centrifuged within 1$2 \mathrm{~min}$ after sampling $\left(2000 \mathrm{~g}\right.$ at $4{ }^{\circ} \mathrm{C}$ for $\left.25 \mathrm{~min}\right)$. Aliquots of plasma were taken from the centre of the sample and stored at $-80^{\circ} \mathrm{C}$ until analysed. Plasma F1 +2 and TAT levels were measured by enzyme immunoassay (Enzygnost F1+2 and Enzygnost TAT micro, Behringwerke AG, Marburg, Germany). Plasma FPA levels were measured by radioimmunoassay (Byk Sangtec Diagnostica) after extraction of fibrinogen with bentonite, according to the manufacturer's instructions.

\section{Other measurements}

Platelet counts, platelet size (median platelet volume) and haematocrit were determined in venous blood samples using a Cellanalyzer CA 460 (Medonic AB, Solna, Sweden). Platelet counts and haematocrit were assessed in EDTA-anticoagulated blood, whereas platelet size was measured in citrated blood [0.12 mol/1, 4:1 (v/v) blood/ citrate], as described previously [19]. Plasma concentrations of thrombin markers during interventions were corrected for changes in the haematocrit; correction factors were calculated from the following formula [20]: 1 - haematocrit intervention $_{\text {/ }}$ - haematocrit ${ }_{\text {rest }}$.

Plasma catecholamine concentrations were determined in samples from blood anticoagulated with EDTA using high-performance cation-exchange liquid chromatography with amperometric detection [21].

\section{Statistics}

Data are presented as means \pm S.E.M. Data on filtragometry and flow cytometry were logarithmically transformed before statistical evaluation because of asymmetrical distribution of the data (for flow-cytometric data $\log [1+x]$ was used since some values were $<1$ ). Paired $t$-tests were used to compare resting and intervention values. $P<0.05$ was considered statistically significant. The statistical software used was Statview (Abacus Concepts Inc., Berkley, CA, U.S.A.).

\section{RESULTS}

\section{Cardiovascular variables, catecholamines in plasma and platelet counts}

The workload achieved during the exercise test was $269 \pm 12 \mathrm{~W}$ (range 200-340 W). Mean exercise time was $23 \pm 1 \mathrm{~min}(16-30 \mathrm{~min})$. As shown in Table 1, physical 
Table I Cardiovascular variables and plasma catecholamines measured after $60 \mathrm{~min}$ rest, after interventions and after $\mathbf{6 0}$ min recovery

Data are presented as means \pm S.E.M. ${ }^{*} P<0.05,{ }^{* *} P<0.01,{ }^{* * *} P<0.001$ (paired $t$-test; compared with rest). During control experiments measurements were performed after 60, 100 and $160 \mathrm{~min}$ respectively. Blood for catecholamine measurements was, for technical reasons, obtained after filtragometry measurements were completed. This resulted in a delay of I to $2 \mathrm{~min}$ and thus underestimation of the peak levels upon termination of exercise. NA, not assessed.

\begin{tabular}{|c|c|c|c|c|}
\hline & $\begin{array}{l}\text { Control } \\
(n=\mathrm{II})\end{array}$ & $\begin{array}{l}\text { Exercise } \\
(n=10)\end{array}$ & $\begin{array}{l}\text { Adrenaline } \\
(n=10)\end{array}$ & $\begin{array}{l}\text { Mental stress } \\
(n=10)\end{array}$ \\
\hline \multicolumn{5}{|c|}{ Heart rate (beats/min) } \\
\hline Rest & $58 \pm 3$ & $58 \pm 2$ & $59 \pm 2$ & $58 \pm 3$ \\
\hline Intervention & $59 \pm 2$ & $193 \pm 3^{* * *}$ & $70 \pm 2^{* * *}$ & $86 \pm 3^{* * *}$ \\
\hline Recovery & $53 \pm 1$ & $71 \pm 2$ & $59 \pm 2$ & $57 \pm 2$ \\
\hline \multicolumn{5}{|c|}{ Systolic blood pressure (mmHg) } \\
\hline Rest & $114 \pm 2$ & $112 \pm 2$ & $119 \pm 2$ & $116 \pm 4$ \\
\hline Intervention & $113 \pm 1$ & $191 \pm 5^{* * *}$ & $143 \pm 2^{* * *}$ & $145 \pm 4^{* * *}$ \\
\hline Recovery & $115 \pm 3$ & $110 \pm 3$ & $119 \pm 2$ & $117 \pm 1$ \\
\hline \multicolumn{5}{|c|}{ Diastolic blood pressure (mmHg) } \\
\hline Rest & $69 \pm 2$ & & $69 \pm 2$ & $68 \pm 2$ \\
\hline Intervention & $72 \pm 2^{* * *}$ & NA & $67 \pm 2$ & $86 \pm 3^{* * *}$ \\
\hline Recovery & $73 \pm 2$ & & $71 \pm 2$ & $71 \pm 3$ \\
\hline \multicolumn{5}{|c|}{ Noradrenaline $(\mathrm{nmol} / \mathrm{l})$} \\
\hline Rest & $1.40 \pm 0.19$ & $1.48 \pm 0.12$ & $1.58 \pm 0.11$ & $1.46 \pm 0.11$ \\
\hline Intervention & $1.46 \pm 0.17$ & $13.56 \pm 1.90^{* * *}$ & $2.00 \pm 0.19^{*}$ & $1.77 \pm 0.16^{*}$ \\
\hline Recovery & $1.40 \pm 0.11$ & $1.74 \pm 0.11$ & $1.72 \pm 0.13$ & $1.35 \pm 0.08$ \\
\hline \multicolumn{5}{|c|}{ Adrenaline (nmol/l) } \\
\hline Rest & $0.10 \pm 0.01$ & $0.14 \pm 0.01$ & $0.14 \pm 0.03$ & $0.19 \pm 0.08$ \\
\hline Intervention & $0.12 \pm 0.01$ & $1.12 \pm 0.25^{* *}$ & $3.34 \pm 0.35^{* * *}$ & $0.38 \pm 0.08^{*}$ \\
\hline Recovery & $0.12 \pm 0.01$ & $0.17 \pm 0.01$ & $0.23 \pm 0.03$ & $0.18 \pm 0.04$ \\
\hline
\end{tabular}

Table 2 Platelet counts and platelet size distribution (median platelet volume) assessed after $60 \mathrm{~min}$ rest, after interventions and after $60 \mathrm{~min}$ recovery

Data are presented as means \pm S.E.M. ${ }^{* *} P<0.00$ I (intervention versus rest; paired $t$-test). During control experiments measurements were performed after 60,100 and 160 min respectively.

\begin{tabular}{lllll}
\hline & $\begin{array}{l}\text { Control } \\
(n=\mathrm{II})\end{array}$ & $\begin{array}{l}\text { Exercise } \\
(n=10)\end{array}$ & $\begin{array}{l}\text { Adrenaline } \\
(n=10)\end{array}$ & $\begin{array}{l}\text { Mental stress } \\
(n=10)\end{array}$ \\
\hline $\begin{array}{l}\text { Platelet counts }\left(10^{9} / \mathrm{l}\right) \\
\quad \text { Rest }\end{array}$ & $241 \pm 10$ & $249 \pm 15$ & $250 \pm 10$ & $241 \pm 10$ \\
$\quad$ Intervention & $249 \pm 13$ & $329 \pm 23^{* * *}$ & $309 \pm 15^{* * *}$ & $246 \pm 12$ \\
$\quad$ Recovery & $249 \pm 12$ & $243 \pm 17$ & $234 \pm 9$ & $234 \pm 9$ \\
$\begin{array}{l}\text { Median platelet volume (fl) } \\
\text { Rest }\end{array}$ & $8.4 \pm 0.1$ & $8.4 \pm 0.2$ & $8.3 \pm 0.1$ & $8.5 \pm 0.2$ \\
$\quad$ Intervention & $8.2 \pm 0.1$ & $8.4 \pm 0.2$ & $8.1 \pm 0.1$ & $8.5 \pm 0.2$ \\
$\quad$ Recovery & $8.3 \pm 0.1$ & $8.4 \pm 0.2$ & $8.2 \pm 0.2$ & $8.4 \pm 0.1$ \\
\hline
\end{tabular}

exercise, adrenaline infusion and mental stress produced the expected effects, with significant but differential increments in heart rate, systolic blood pressure and plasma catecholamines; these variables remained stable during time control experiments.

Platelet counts in venous blood increased significantly during exercise and adrenaline infusion (Table 2); there was no significant increase during mental stress. Platelet size (assessed as median platelet volume) was not affected by any of the stressors.

\section{Filtragometry ex vivo}

Filtragometry responses to the interventions are shown in Figure 1. Physical exercise and adrenaline infusion increased platelet aggregability significantly; the platelet- 

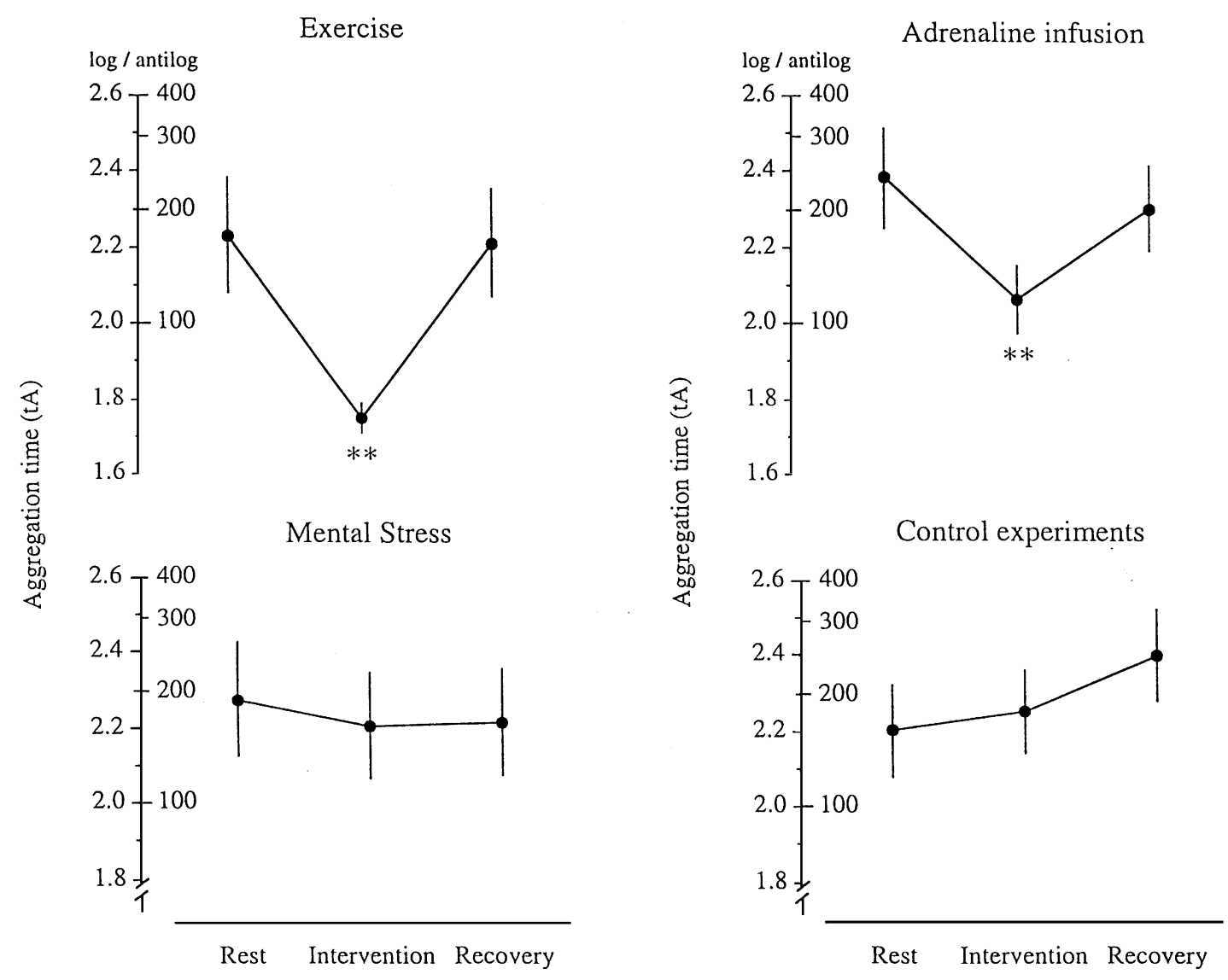

Figure I Platelet aggregability (filtragometry ex vivo) before, during and $60 \mathrm{~min}$ after exercise $(n=10)$, adrenaline infusion $(n=10)$ and mental stress $(n=10)$, and during control experiments $(n=I I)$

A decreased aggregation time reflects increased aggregability. Values are means \pm S.E.M. Significance levels are derived from paired $t$-test (intervention versus rest; $\left.{ }^{* *} P<0.01\right)$.

activating effects were reversible and more pronounced during exercise. Mental stress had no significant effects. In control experiments, platelet aggregability tended to decrease over time (Figure 1).

\section{Flow cytometry of platelets in whole blood}

\section{Unstimulated samples}

In unstimulated samples from resting subjects, platelet fibrinogen binding was low (Table 3), as was the percentage of platelets binding fibrinogen (results not shown), indicating adequate sampling and sample handling. The percentage of platelets expressing GPIb $\alpha$ and GPIIb-IIIa was $99.5 \pm 0.2$ (S.D.) for both parameters.

After exercise, there was a slight but significant increase in fibrinogen binding, and a decrease in GPIb $\alpha$ expression. Both parameters returned to baseline levels $60 \mathrm{~min}$ later. However, similar findings were seen for resting fibrinogen binding during control experiments. There were no significant effects of adrenaline infusion on fibrinogen binding or GPIb $\alpha$ expression in unstimulated samples. During mental stress, fibrinogen binding index and GPIb $\alpha$ expression were not significantly altered (Table 3), but there was a small significant increase in the percentage of cells positive for fibrinogen (from $1.05 \pm 0.08 \%$ at rest to $1.28 \pm 0.10 \%$ during mental stress; $P<0.05)$.

There was no difference in the expression of GPIIbIIIa with any of the interventions (results not shown).

Platelets from one subject in the mental stress experiments had very high fibrinogen binding during mental stress and after $60 \mathrm{~min}$ of recovery $(7.0 \%$ positive during stress and $12.2 \%$ positive at recovery). Flow-cytometric data from this outlier were more than 3 S.D. from the mean of the other subjects, and were therefore excluded from the group analysis.

\section{Agonist-stimulated samples}

Fibrinogen binding in response to thrombin stimulation was increased by exercise (Figure 2). The effect was evident at mid-range and high concentrations of thrombin (Figure 2). Similar increases in thrombin-induced fibrinogen binding were seen after adrenaline infusion (Figure 2). Mental stress increased thrombin-induced 
Table 3 Data on fibrinogen and GPIb binding indices in unstimulated whole blood samples Values are means \pm S.E.M. Statistical evaluation was performed by paired $t$-test (intervention versus rest; ${ }^{*} P<0.05$, $\left.{ }^{* *} P<0.01 ; n=9-1 \mathrm{I}\right)$.

\begin{tabular}{lllll}
\hline & Control & Exercise & Adrenaline & Mental stress \\
\hline Fibrinogen binding index & & & & \\
$\quad$ Rest & $0.026 \pm 0.002$ & $0.028 \pm 0.002$ & $0.038 \pm 0.010$ & $0.031 \pm 0.004$ \\
$\quad$ Intervention & $0.035 \pm 0.002^{* *}$ & $0.036 \pm 0.002^{*}$ & $0.031 \pm 0.003$ & $0.037 \pm 0.003$ \\
$\quad$ Recovery & $0.030 \pm 0.005$ & $0.029 \pm 0.008$ & $0.033 \pm 0.005$ & $0.029 \pm 0.003$ \\
GPIb binding index & & & & \\
$\quad$ Rest & $23.47 \pm 1.39$ & $28.59 \pm 1.29$ & $22.96 \pm 0.82$ & $25.34 \pm 1.07$ \\
$\quad$ Intervention & $23.37 \pm 1.28$ & $27.75 \pm 1.30^{*}$ & $22.76 \pm 0.86$ & $25.09 \pm 1.05$ \\
$\quad$ Recovery & $23.38 \pm 1.33$ & $28.53 \pm 1.42$ & $22.37 \pm 0.91$ & $25.18 \pm 1.04$ \\
\hline
\end{tabular}
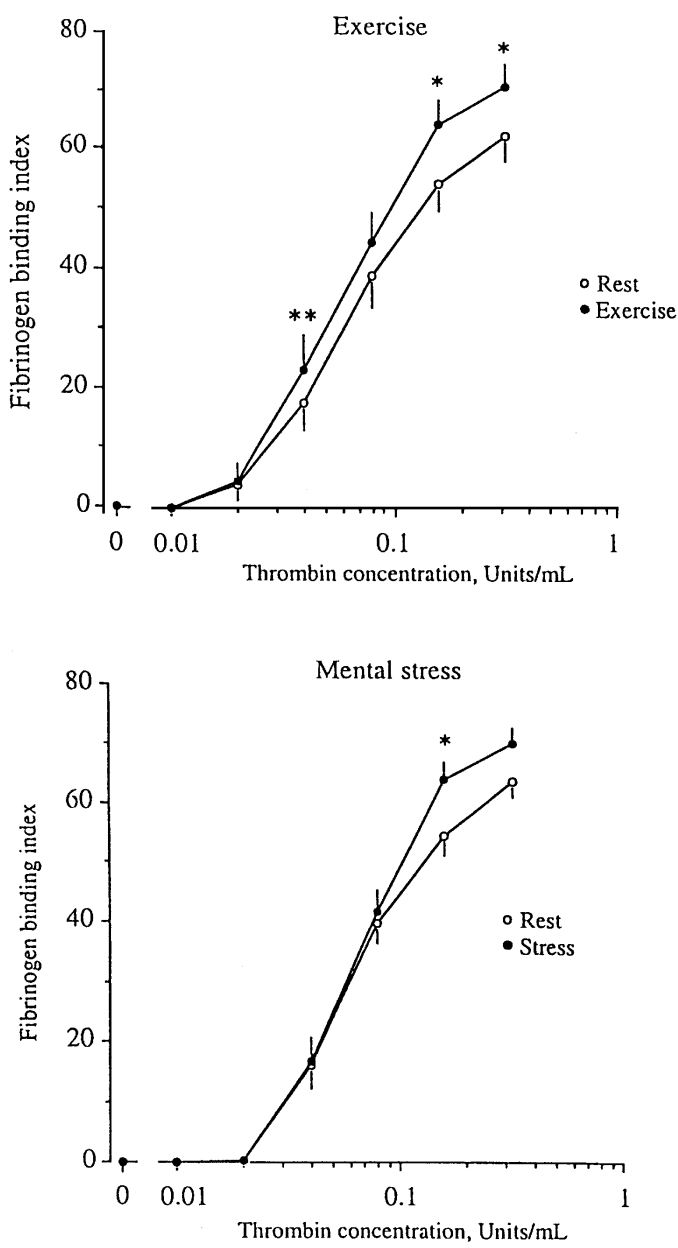
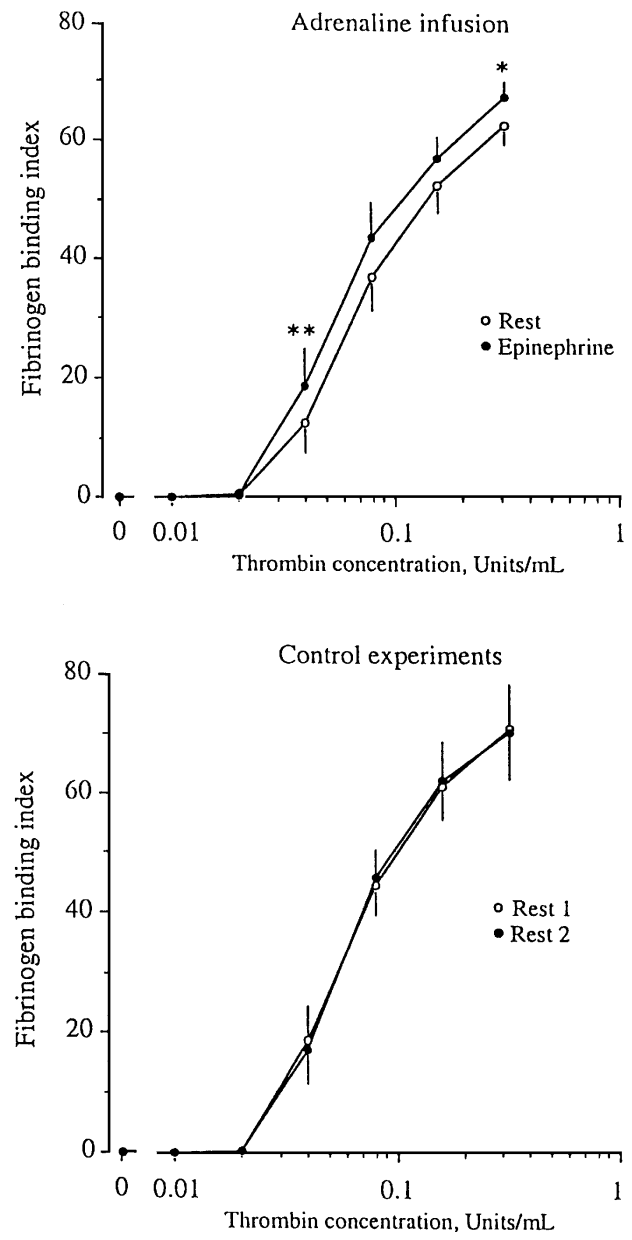

Figure 2 Thrombin-induced fibrinogen binding (binding index) in whole blood before and during exercise $(n=10)$, adrenaline (epinephrine) infusion $(n=10)$ and mental stress $(n=9)$, and during control experiments $(n=I I)$

Platelet fibrinogen binding, a prerequisite for platelet aggregation, is expressed as binding index and calculated from the percentage of positive cells and mean fluorescence intensity. Data are presented as means \pm S.E.M. Significance levels are derived from paired $t$-test (intervention versus rest; ${ }^{*} P<0.05,{ }^{* *} P<0.01$ ).

fibrinogen binding at high concentrations of thrombin $(\geqslant 0.16$ units $/ \mathrm{ml})$, whereas no effects were seen at lower concentrations. Thrombin sensitivity did not change during the control experiments.
ADP-induced fibrinogen binding was enhanced by exercise and by adrenaline infusion, in line with previous findings [11], but no change in ADP sensitivity was seen during mental stress (results not shown). 
Table 4 Thrombin generation (FI +2 and TAT) and thrombin activity (FPA)

Values are means \pm S.E.M. ${ }^{*} P<0.05$ (intervention versus rest; paired $t$-test).

\begin{tabular}{|c|c|c|c|c|}
\hline & $\begin{array}{l}\text { Control } \\
(n=\mathrm{II})\end{array}$ & $\begin{array}{l}\text { Exercise } \\
(n=10)\end{array}$ & $\begin{array}{l}\text { Adrenaline } \\
(n=10)\end{array}$ & $\begin{array}{l}\text { Mental stress } \\
(n=10)\end{array}$ \\
\hline \multicolumn{5}{|l|}{$\mathrm{FI}+2(\mathrm{nmol} / \mathrm{l})$} \\
\hline Rest & $0.65 \pm 0.04$ & $0.70 \pm 0.07$ & $0.74 \pm 0.05$ & $0.58 \pm 0.07$ \\
\hline Intervention & $0.62 \pm 0.04$ & $0.81 \pm 0.07^{*}$ & $0.71 \pm 0.06$ & $0.55 \pm 0.06$ \\
\hline Recovery & $0.62 \pm 0.04$ & $0.75 \pm 0.04$ & $0.65 \pm 0.05$ & $0.54 \pm 0.06$ \\
\hline \multicolumn{5}{|l|}{ TAT (ng/ml) } \\
\hline Rest & $1.18 \pm 0.19$ & $0.76 \pm 0.15$ & $0.93 \pm 0.09$ & $0.87 \pm 0.08$ \\
\hline Intervention & $0.99 \pm 0.09$ & $1.14 \pm 0.11^{*}$ & $1.14 \pm 0.12^{*}$ & $1.16 \pm 0.14$ \\
\hline Recovery & $1.17 \pm 0.12$ & $1.21 \pm 0.18$ & $1.05 \pm 0.12$ & $0.99 \pm 0.11$ \\
\hline \multicolumn{5}{|l|}{ FPA (ng/ml) } \\
\hline Rest & $0.72 \pm 0.05$ & $0.61 \pm 0.05$ & $1.48 \pm 0.19$ & $1.48 \pm 0.22$ \\
\hline Intervention & $1.08 \pm 0.28$ & $1.28 \pm 0.28^{*}$ & $1.56 \pm 0.31$ & $1.31 \pm 0.17$ \\
\hline Recovery & $1.09 \pm 0.27$ & $0.98 \pm 0.18$ & $2.38 \pm 0.70$ & $2.05 \pm 0.46$ \\
\hline
\end{tabular}

\section{Thrombin markers}

Data on the effects of the interventions on thrombin generation (F1+2 and TAT levels in plasma) and thrombin activity (FPA levels in plasma) are summarized in Table 4. Thrombin generation and thrombin activity were unchanged during control experiments.

Thrombin generation and thrombin activity increased in response to physical exercise, as shown by elevated plasma levels of $\mathrm{F} 1+2$, TAT and FPA. At recovery $\mathrm{F} 1+2$ levels returned to baseline, whereas TAT levels remained elevated. Plasma FPA tended to return towards baseline at recovery (Table 4).

During adrenaline infusion, TAT levels increased significantly, whereas F1 + 2 levels were unchanged. TAT levels returned towards baseline values at recovery, whereas F1 +2 levels were significantly lower at recovery compared with during the infusion.

Mental stress did not influence F1 +2 levels, but tended to increase TAT $(P=0.08)$. FPA levels were not significantly altered during or after mental stress or adrenaline infusion.

\section{DISCUSSION}

The present study shows that haemostasis can be activated by various external stressors. Increased platelet sensitivity to thrombin, as well as increased formation of thrombin, was observed immediately after heavy physical exercise and during adrenaline infusion. Furthermore, platelet aggregability, as measured by filtragometry ex vivo, was enhanced. There were also indications of mild activation of haemostasis during mental stress, with slight enhancement of thrombin-induced fibrinogen binding at high thrombin concentrations, and tendencies towards increased thrombin formation. The patterns of haemostatic activation induced by physical exercise, adrenaline infusion and mental stress are thus similar, but the magnitudes of the responses differ, physical exercise having the most pronounced effects.

There was some discrepancy between the thrombin generation markers with respect to effects of the stressors. Exercise elevated both TAT and F1 +2 levels in plasma, but adrenaline infusion and mental stress had less clearcut effects. TAT, but not $\mathrm{F} 1+2$ levels, increased during adrenaline infusion and tended to increase during mental stress $(P=0.08)$. The reason for this discrepancy is unclear. Theoretically, responses to short-lasting stressors should be more easily revealed by measurements of TAT, which has a half-life of approximately $3 \mathrm{~min}$ [22], compared with $\mathrm{F} 1+2$, which has a half-life of approximately $90 \mathrm{~min}$ [23]. Changes in the elimination of the thrombin markers, as well as alterations of antithrombin and prothrombin levels during the interventions, may also have had some influence. TAT appears to be a more sensitive indicator of thrombin generation. Thus, patients with non-valvular atrial fibrillation had elevated levels of TAT but not F1 +2 [24], and patients with proximal deep venous thrombosis showed different patterns for TAT and F1+2, with more clear-cut increments in the former [25]. Our findings of elevated TAT levels during adrenaline infusion and, to a lesser degree, during mental stress support the contention that TAT is a more sensitive marker of ongoing thrombin generation than F1+2. Significantly increased thrombin activity was observed only during exercise. Thus, exercise is a more powerful activator of coagulation than mental stress or adrenaline infusion.

The present flow-cytometric data show that single platelets have an increased sensitivity to thrombin after exercise and adrenaline infusion, and to some extent during mental stress. The dose-response curves for thrombin-induced platelet fibrinogen binding were shifted upwards during the interventions, and these 
effects were most evident at mid-range and high concentrations of thrombin. Thrombin is known to induce surface expression of internal pools of the fibrinogen receptor [26]. Sensitization of platelets by sympathoadrenal activation may enhance such an effect of thrombin stimulation in vitro, and thus lead to enhanced fibrinogen binding. However, the GPIIb-IIIa expression of unstimulated platelets was not influenced by the stressors studied.

The expression of GPIb $\alpha$ in unstimulated samples was significantly reduced after exercise, but no significant changes in GPIb $\alpha$ were noted during the other interventions. It has been suggested that the GPIb-IX complex is internalized after agonist stimulation [27]. In view of this, our present observation further supports the idea that physical exercise activates platelets in vivo. Another possibility that should be considered is that reduced GPIb $\alpha$ expression may reflect proteolysis of the GPIb $\alpha$ receptors $[28,29]$; exercise may lead to leucocyte activation with release of proteolytic enzymes leading to the removal of the GPIb $\alpha$ N-terminal section [30]. Further studies are needed to resolve this issue. Our findings are nonetheless in line with those of Kestin et al. [31], who observed reduced expression of GPIb and increased platelet sensitivity to thrombin after vigorous exercise in healthy subjects.

Previously we have found that mental stress evoked by the present colour word test has platelet-activating effects in young healthy males [3]. However, in healthy elderly subjects, this phenomenon could not be observed, but platelet responses to mental stress tended to be more pronounced in a population with angina pectoris [5]. In the present study, there were only weak indications of haemostatic activation during mental stress. However, the present study is small. A larger study would be required to fully establish these effects of mental stress on haemostasis. It should also be noted that there is considerable interindividual variation of responses to stressful situations.

The mechanisms behind the haemostatic activation observed in response to the various 'stressors' are unknown. Catecholamine-induced sensitization of platelets is probably important $[3,11,32]$. Elevations of plasma adrenaline or noradrenaline by intravenous infusions elicit platelet activation $[3,6,7]$. It should be emphasized, however, that neither exercise nor mental stress elevates plasma adrenaline to levels high enough to have platelet-activating effects in vivo, whereas noradrenaline has clear-cut platelet-activating effects at, or below, the plasma levels obtained in the present exercise study (i.e. around 10-15 nmol/l) [7]. Noradrenaline is thus more important than adrenaline for exercise-induced platelet activation. Shear-induced platelet activation [33], due to a hyperdynamic circulation, and release of ADP from erythrocytes and platelets [34], may contribute to the effects of exercise, adrenaline infusion and mental stress. It is of interest to note that exercise, which caused the most pronounced haemodynamic effects, was associated with the strongest platelet-activating effects. Enhanced formation of thrombin may also have contributed to the platelet activation, as we observed enhanced thrombin generation, as well as increased platelet sensitivity to thrombin. Studies of platelet responses to exercise in the presence of thrombin inhibition, however, are needed to establish this mechanism.

Thrombocytosis was observed during exercise and adrenaline infusion. A slight, but non-significant increase in platelet counts was also noted during mental stress. These are well-known phenomena and related to alterations in splenic blood flow in response to sympathoadrenal activation (see [6] and references therein). From a mechanistic point of view, thrombocytosis is of interest as it may enhance platelet aggregate formation by increasing platelet-platelet interactions.

In conclusion, heavy physical exercise and high physiological levels of adrenaline increase platelet aggregability and enhance platelet sensitivity to thrombin and thrombin generation - effects which are more pronounced during exercise. Mental stress has similar but weaker effects. Thus, activities associated with sympathoadrenal activation may have prothrombotic effects, partly through the enhancement of thrombin formation and partly due to increased platelet sensitivity to thrombin. Such mechanisms may be involved in the triggering of coronary artery disease, especially when they occur in combination with impaired fibrinolytic responsiveness, as shown recently in patients with angina pectoris [35].

\section{ACKNOWLEDGMENTS}

The technical assistance of Maud Daleskog and MajChristina Johansson is gratefully acknowledged. Martin Åhlenius is acknowledged for statistical services. The study was supported by grants from the Swedish Heart Lung Foundation, the Swedish Medical Research Council (5930), the Swedish Society of Medicine, the Salus 60year fund, King Gustav V and Queen Victoria's foundation and the Karolinska Institute.

\section{REFERENCES}

1 Willich, S. N., Maclure, M., Mittleman, M., Arntz, H-R. and Muller, J. E. (1993) Sudden cardiac death. Support for a role of triggering in causation. Circulation 87, 1442-1450

2 Muller, J. E., Abela, G. S., Nesto, R. W. and Tofler, G. (1994) Triggers, acute risk factors and vulnerable plaques: the lexicon of a new frontier. J. Am. Coll. Cardiol. 23, 809-813

3 Hjemdahl, P., Larsson, P. T. and Wallén, N. H. (1991) Effects of stress and beta-blockade on platelet function. Circulation 84 (Suppl.), VI44-VI61

4 Hjemdahl, P. (1995) Platelet reactivity, exercise and stable coronary heart disease. Eur. Heart J. 16, 1017-1019 
5 Wallén, N. H., Held, C., Rehnqvist, N. and Hjemdahl, P. (1997) Effects of mental and physical stress on platelet function in patients with stable angina pectoris and healthy controls. Eur. Heart J. 18, 807-815

6 Larsson, P. T., Wallén, N. H., Egberg, N. and Hjemdahl, P. (1992) Alpha-adrenoceptor blockade by phentolamine inhibits adrenaline-induced platelet activation in vivo without affecting resting measurements. Clin. Sci. 82, 369-376

7 Larsson, P. T., Wallén, N. H. and Hjemdahl, P. (1994) Norepinephrine-induced human platelet activation in vivo is only partly counteracted by aspirin. Circulation $\mathbf{8 9}$, 1951-1957

8 Herren, T., Bärtsch, P., Haeberli, A. and Straub, P. W. (1992) Increased thrombin-antithrombin III complexes after $1 \mathrm{~h}$ of physical exercise. J. Appl. Physiol. 73, 2499-2504

9 Prisco, D., Paniccia, R., Guarnaccia, V. et al. (1993) Thrombin generation after physical exercise. Thromb. Res. 69, 159-164

10 Borg, G. A. V. (1982) A category scale with properties for intermodal and interindividual comparisons. In Psychophysical Judgement and the Process of Perception (Geissler, H. G. and Petzolds, P., eds.), pp. 25-34, VEB Deutcher Verlag der Wissenschaften, Berlin

11 Hjemdahl, P., Chronos, N. A. F., Wilson, D. J., Bouloux, P. and Goodall, A. H. (1994) Epinephrine sensitizes human platelets in vivo and in vitro as studied by fibrinogen binding and P-selectin expression. Arterioscler. Thromb. 14, 77-84

12 Frankenheuser, M., Mellis, I., Rissler, A., Björkvall, C. and Patkai, P. (1968) Catecholamine excretion as related to cognitive and emotional reaction patterns. Psychosom. Med. 30, 109-120

13 Freyschuss, U., Hjemdahl, P., Juhlin-Dannfelt, A. and Linde, B. (1988) Cardiovascular and sympathoadrenal responses to mental stress: influence of beta-blockade. Am. J. Physiol. 255, H1443-H1451

14 Hornstra, G. and ten Hoor, F. (1975) The filtragometer: a new device for measuring platelet aggregation in venous blood of man. Thromb. Diath. Haemorrh. 34, 531-544

15 Larsson, P. T., Hjemdahl, P., Olsson, G., Egberg, N. and Hornstra, G. (1989) Altered platelet function during mental stress and adrenaline infusion in humans: evidence for an increased aggregability in vivo as measured by filtragometry. Clin. Sci. 76, 369-376

16 Goodall, A. H. (1991) Platelet activation during preparation and storage: detection by flow cytometry. Blood Coagul. Fibrinolysis 2, 377-382

17 Janes, S. L., Wilson, D. J., Chronos, N. and Goodall, A. H. (1993) Evaluation of whole blood flow cytometric detection of platelet bound fibrinogen on normal subjects and patients with activated platelets. Thromb. Haemost. 70, 659-666

18 Michelson, A. D. (1994) Platelet activation by thrombin can be directly measured in whole blood through the use of the peptide GPRP and flow cytometry: methods and clinical applications. Blood Coagul. Fibrinolysis 5, 121-131

19 Bath, P. M. V. (1993) The routine measurement of platelet size using sodium citrate alone as the anticoagulant. Thromb. Haemost. 70, 687-690

20 Nguyen, G., Horellou, M. H., Kruithof, E. K. O., Conard, J. and Samama, M. M. (1988) Residual plasminogen activator inhibitor activity after venous stasis as a criterion for hypofibrinolysis: a study in 83 patients with confirmed deep vein thrombosis. Blood 72, 601-605

21 Hjemdahl, P. (1987) Catecholamine measurements in plasma by high-performance liquid chromatography with electrochemical detection. Methods Enzymol. 142, 521-534

22 Shifman, M. A. and Pizzo, S. V. (1982) The in vivo metabolism of antithrombin III and antithrombin complexes. J. Biol. Chem. 257, 3243-3248

23 Bauer, K. A., Goodman, T. L., Kass, B. L. and Rosenberg, R. D. (1985) Elevated factor Xa activity in the blood of asymtomatic patients with congenital antithrombin deficiency. J. Clin. Invest. 76, 826-836

24 Mitusch, R., Siemens, H. J., Garbe, M., Wagner, T. Sheikhzadeh, A. and Diederich, K. W. (1996) Detection of a hypercoagulable state in nonvalvular atrial fibrillation and the effect of anticoagulant therapy. Thromb. Haemost. $75,219-223$

25 Elias, A., Bonfils, S., Daoud-Elias, M. et al. (1993) Influence of long term anticoagulants upon prothrombin fragment $1+2$, thrombin-antithrombin III complex and $\mathrm{D}$-dimer levels in patients affected by proximal deep vein thrombosis. Thromb. Haemost. 69, 302-305

26 Woods, V. L., Wolff, L. E. and Keller, D. M. (1986) Resting platelets contain a substantial centrally located pool of glycoprotein IIb-IIIa complex which may be accessible to some but not other extracellular proteins. J. Biol. Chem. 261, 15242-15251

27 Michelson, A. D., Ellis, P. A., Barnard, M. R., Matic, G. B., Viles, A. F. and Kestin, A. S. (1991) Downregulation of the platelet surface glycoprotein Ib-IX complex in whole blood stimulated by thrombin, adenosine diphosphate, or an in vivo wound. Blood 77, 770-779

28 Nurden, A. T. (1994) Human platelet membrane glycoproteins. In Haemostasis and Thrombosis (Bloom, A. L., Forbes, C. D., Thomas, D. C. and Tuddenham, E. G. D., eds.), pp. 115-165, Churchill Livingstone, Edinburgh

29 LaRosa, C. A., Rohrer, M. J., Benoit, S. E., Barnard, M. R. and Michelson, A. D. (1994) Neutrophil cathepsin G modulates the platelet surface expression of the glycoprotein (GP) Ib-IX complex by proteolysis of the von Willebrand factor binding site on GPIb alpha and by a cytoskeletal-mediated redistribution of the remainder of the complex. Blood 84, 158-168

30 Camus, G., Deby-Dupont, G., Duchateau, J., Deby, C., Pincemail, J. and Lamy, M. (1994) Are similar inflammatory factors involved in strenuous exercise and sepsis? Intensive Care Med. 20, 602-610

31 Kestin, A. S., Ellis, P. A., Barnard, M. R., Errichetti, A. Rosner, B. A. and Michelson, A. D. (1993) Effect of strenous exercise on platelet activation state and reactivity. Circulation 88, 1502-1511

32 Ardlie, N. G., McGuiness, J. A. and Garrett, J. J. (1985) Effect on human platelets of catecholamines at levels achieved in the circulation. Atherosclerosis 58, 251-259

33 O’Brien, J. R. (1990) Shear-induced platelet aggregation. Lancet 335, 711-713

34 Moritz, M. W., Reimers, R. C. and Baker, R. K. (1983) Role of cytoplasmic and releasable ADP in platelet aggregation induced by laminar shear stress. J. Lab. Clin. Med. 101, 537-544

35 Held, C., Hjemdahl, P., Rehnqvist, N. et al. (1997) Fibrinolytic variables and cardiovascular prognosis in patients with stable angina pectoris, treated with verapamil or metoprolol. Circulation 95, 2380-2386 